\title{
8
}
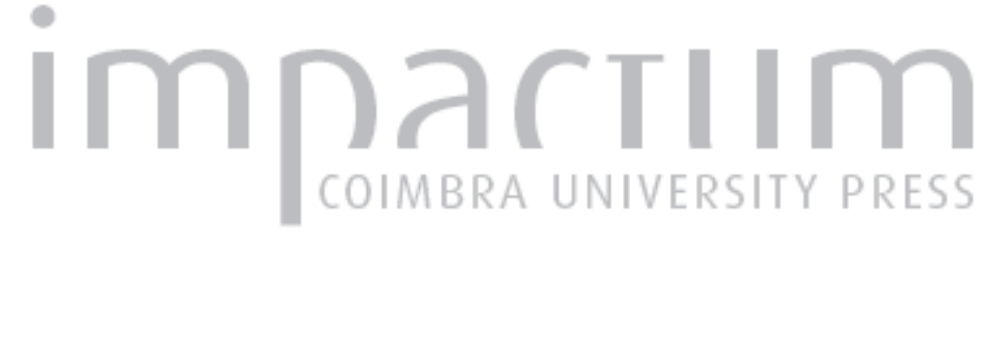

\section{L'administration du territoire vetton à l'époque romaine: statuts juridiques et institutions}

\author{
Autor(es): $\quad$ Bonnaud, Christophe
}

Publicado por: Imprensa da Universidade de Coimbra

URL persistente:

URI:http://hdl.handle.net/10316.2/45357

DOI:

DOI:https://dx.doi.org/10.14195/1647-8657_40_1

Accessed : $\quad$ 26-Apr-2023 05:13:36

A navegação consulta e descarregamento dos títulos inseridos nas Bibliotecas Digitais UC Digitalis, UC Pombalina e UC Impactum, pressupõem a aceitação plena e sem reservas dos Termos e Condições de Uso destas Bibliotecas Digitais, disponíveis em https://digitalis.uc.pt/pt-pt/termos.

Conforme exposto nos referidos Termos e Condições de Uso, o descarregamento de títulos de acesso restrito requer uma licença válida de autorização devendo o utilizador aceder ao(s) documento(s) a partir de um endereço de IP da instituição detentora da supramencionada licença.

Ao utilizador é apenas permitido o descarregamento para uso pessoal, pelo que o emprego do(s) título(s) descarregado(s) para outro fim, designadamente comercial, carece de autorização do respetivo autor ou editor da obra.

Na medida em que todas as obras da UC Digitalis se encontram protegidas pelo Código do Direito de Autor e Direitos Conexos e demais legislação aplicável, toda a cópia, parcial ou total, deste documento, nos casos em que é legalmente admitida, deverá conter ou fazer-se acompanhar por este aviso.

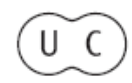


UNIVERSIDADE DE COIMBRA

FACULDADE DE LETRAS

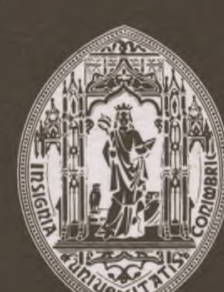

CONIMBRIGA

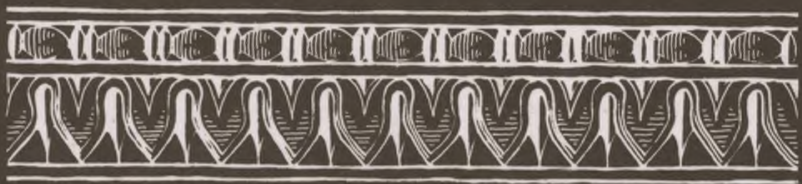

VOLUME XL - 2001 


\section{Christophe Bonnaud}

Professeur d'Histoire au Lycée Français du Caire; docteur en Histoire Ancienne.

\section{L'ADMINISTRATION DU TERRITOIRE VETTON À L'ÉPOQUE ROMAINE - STATUTS JURIDIQUES ET INSTITUTIONS \\ "Conimbriga" XL (2001) p. 5-36}

Résumé: Dans l'Antiquité, l'ouest de la Meseta espagnole ainsi qu'une mince frange du Portugal étaient occupés par les Vettons, population sans doute d'origine celtique qui s'insère dans la vaste nébuleuse des peuples protohistoriques indo-européens de la péninsule ibérique. Les Vettons ont été intégrés dans l'orbite romaine dans la deuxième moitié de Ilème siècle av. J.C., dans le contexte des guerres lusitaniennes, avant d'être inclus dans la province de Lusitanie, et plus tard dans le conventus de Mérida. Alors que la plus grande partie de ce conventus était peuplée de Lusitaniens, l'intégration des Vettons dans les structures administratives romaines est loin d'avoir été négligée par les autorités romaines. Ainsi, on a pu constater la volonté romaine de rassembler les Vettons à l'intérieur des limites de la Lusitanie. De même, à certaines périodes de l'histoire administrative de la province, les Vettons semblent avoir été l'objet d'une attention particulière de la part des autorités comme en témoigne l'existence de fonctionnaires attachés à la «provincia Lusitaniae et Vettoniae». Enfin, le territoire vetton, malgré la faiblesse de sa vie urbaine, n'a pas été écartée du phénomène municipal, comme l'attestent un certain nombre de sources épigraphiques qui nous permettent d'appréhender l'armature administrative, très hétérogène, du territoire vetton et les statuts juridiques de ses communautés.

Resumo: Na Antiguidade, a parte ocidental da Meseta espanhola, assim como uma estreita faixa de Portugal estavam ocupadas pelos Vetoes, povo

Conimbriga, 40 (2001) 5-35 
sem dúvida de origem céltica que se enquadra na vasta nebulosa dos povos proto-históricos indo-europeus, da Península Ibérica.

Os Vetoes foram integrados na órbita romana no decorrer da segunda metade do século II a. C., no contexto das guerras dos Lusitanos, antes de serem incluídos na província da Lusitânia e, mais tarde, no conventus de Mérida.

Ainda que a maior parte desse conventus estivesse povoada por Lusitanos, a integração dos Vetoes nas estruturas administrativas romanas esteve longe de ser negligenciada pelas autoridades romanas. Desta sorte, pôde atestar-se a vontade dos Romanos de juntarem os Vetoes no interior dos limites da Lusitânia. Por outro lado, em determinados períodos da história administrativa da província, os Vetoes parecem ter sido objecto de particular atenção por parte das autoridades, como o atesta a existência de funcionários ligados à «provinda Lusitaniae et Vettoniae».

Finalmente, o território vetão, apesar da sua débil vida urbana, não foi afastado do fenómeno municipal, como documentam um certo número de fontes epigráficas que nos permitem apreender a orgânica administrativa, muito heterogénea, do território vetão e o estatuto jurídico das suas comunidades. 


\section{L'ADMINISTRATION DU TERRITOIRE VETTON A L'EPOQUE ROMAINE: STATUTS JURIDIQUES ET INSTITUTIONS i}

Dans l'Antiquité romaine, la partie orientale du conventus de Mérida était occupée par une population souvent qualifiée de celtique dans Thistoriographie: les Vettons ${ }^{1}{ }^{2}$. Le territoire de ces derniers n'a jamais pu être délimité avec précision, faute de sources suffisantes, mais on sait qu'il s'étendait sur la plus grande partie des provinces de Salamanque et d'Avila, la moitié orientale de celle de Cáceres, avec des appendices sur les provinces de Zamora, Tolède et Badajoz ainsi que sur une frange orientale du Portugal. L'identité culturelle de cette population est également difficile à établir en raison des lacunes des sources et du caractère peu homogène du peuplement de ces terres de Castille et d'Estrémadure. Néanmoins, on sait que la Vettonnie était une terre de castros, sites fortifiés, et surtout de verracos, imposantes sculptures zoomorphes dont la chronologie et les fonctions sont encore source de nombreuses interrogations. Peuple éleveur et fortement marqué par le nomadisme, les Vettons, malgré leurs productions céramiques et métallurgiques apparaissent, à la lumière de l'archéologie, comme un peuple protohistorique connaissant un faible développement socio-économique et subissant fortement les influences des peuples voisins (Astures, Lusitaniens...), y compris au moment des guerres de conquêtes (Ilème siècle av. J.C.). Ainsi, une fois la pacification achevée, les Romains ont dû procéder à l'intégration administrative de ce territoire inclus dans la province de Lusitanie. Comment cette intégration s'est-elle effectuée? Y-a-t-il eu une politique parti-

1 Cet article reprend des passages de notre thèse soutenue à Poitiers le 2 septembre 1999: VETTONIA ANTIQVA. PEUPLEMENT ET SOCIETE DANS L'OUEST DE LA MESETA (5 volumes).

2 On notera ici la parution récente d'un livre sur les Vettons (à l'époque protohistorique): J .R. ÁLVAREZ-S ANCHIS, Los Vettones (BAH, I), Madrid, 1999. 
culière menée par les Romains envers les Vettons en ce domaine? Comment s'articulait la gestion administrative de l'espace vetton, notamment au niveau des différentes cités et en tenant compte de l'évolution juridique que ces dernières ont pu connaître?

\section{L'INTEGRATION DANS LA PROVINCE DE LUSITANIE}

\section{De V Ulterior à la Lusitanie}

De la soumission effective des Vettons (pas avant 132 av. J.C.) à leur intégration dans le système provincial augustéen, soit pendant plus d'une centaine d'années, la Vettonnie a dû être l'objet de profondes mutations: changements d'ordre matériel entraînés par la conquête ${ }^{3}$ (destruction de castros, nouvelles implantations, construction d'un réseau de voies ...), naissance de relations d'un type nouveau entre les Vettons et les Romains, modifications dans l'assise territoriale des Vettons, mais aussi intégration de ces derniers dans un cadre administratif destiné à un meilleur contrôle de cette population et à une meilleure gestion de leur territoire.

Il apparaît que dans un premier temps, les Romains ont fait en sorte d'éliminer des germes de futures tensions. Cette politique de préparation à une meilleure intégration administrative et sociale a sans doute consisté à faire descendre des montagnes lusitaniennes toutes proches ( Mons Herminius), peut-être dès le Ilème siècle avJ.C. et / ou à l'époque de $\mathrm{César}^{4}$, des populations qui ont dû se fondre plus ou

3 Sur la conquête de la Vettonnie, nous renvoyons à notre Vettonia Antiqua. Peuplement et société dans Гouest de la Meseta, tome 2, livre II, pp.33-47. Rappelons ici brièvement que les sources (Tite-Live et Appien essentiellement) évoquent assez peu les Vettons dans le contexte des guerres de conquête. La première mention des Vettons concerne des opérations de police effectuées par les Romains en -193; par la suite, les Vettons apparaissent associés aux Lusitaniens ou bien sont passés sous silence. Cette «discrétion» des Vettons dans les textes doit s'expliquer par une utilisation générique du terme de Lusitaniens qui accaparent l'intérêt des historiens grécolatins en raison de leur rôle initiateur et prépondérant lors des guerres «lusitaniennes».

4 En 137 av. J.C., Galiciens et Lusitaniens furent vaincus par D. Iunius Brutus qui procéda ensuite à des remaniements territoriaux en Estrémadure: J. de Francisco MARTín, Conquista y romanización de Lusitania, Salamanque, 1989, pp. 70-71,83.; DıoN CAssius, XXXVII, 52-53, nous apprend que César, en 61 av. J.C., voulut obliger les Lusitaniens du Mons Herminius à descendre dans la plaine, ce qui déclencha la guerre. 
moins avec les Vettons, d'où la difficulté à différencier l'onomastique vettonne et l'onomastique lusitanienne du Haut-Empire 5 ou à distinguer, dans cette région, un faciès archéologique vetton différent du faciès lusitanien. Ainsi, les régions, entre Tage et Guadiana, de Coria, Alcántara, Cáceres étaient à la fois des zones frontalières sud-occidentales de la Vettonnie, et des régions de peuplement hétérogène ${ }^{6}$. Cette mixité a également pu résulter d'un certain brassage de populations effectué au moment des guerres lusitaniennes, au cours desquelles nous entr'apercevons une «solidarité» vettonne envers les Lusitaniens ${ }^{7}$.

Ainsi, il est difficile de préciser l'origine ethnique de la communauté indigène d'Alcàntara qui, en 104 av. J.C., conclut une deditio avec les Romains. Qu'il s'agisse d'une communauté lusitanienne ou vettonne, nous avons là un exemple concret des conditions dans lesquelles ces populations ont pu se soumettre:

C(aio) Mario, C(aio) Flauio [co(n)s(ulibus)], I L(ucio) Caesio C(aii) fiilio) imperatore, populus Seano[-I I dedit. L. Caesius C(aii) f(ilius) imperator postquam [-] I accepit ad consilium retold (sic) quid eis im[perare?] I censerent de consili(i) sententia imperau[it \ I captiuos equos equas quas cepis(s)ent [-] I omnia dederunt deinde eos L(ucius) Caesius C(aii) If(ilius)] I esse iussit agros et aedificia leges cete[ra] I quae sua fuissent pridie quam se dedid[erunt I - ] extarent eis redidit dum populu[s| I Roomanus (sic) uellet deque ea re eos I eire iussit legatos Cren[-] I Areo Cantoni fiilio) legates (sic).

5 M.L. Albertos Firmat, Onomastique personnelle indigène de la Péninsule Ibérique sous la domination romaine, dans $A N R W$, II, 29, 1983, pp.869-872.

6 II n'existe par ailleurs dans cette région aucun élément naturel susceptible de faire office, sous le Haut-Empire, de limite ethnique entre le Tage et la sierra de Gata, soit le nord-ouest de la province de Cáceres.

7 Appien, Iber., LVI, LVII, LVIII; sur l'identité et la provenance des «Lusitaniens» en guerre contre les Romains, $c f$. L. Pérez Vilatela, Procedencia geográfica de los Lusitanos de las guerras del siglo II a.C. en los autores clásicos (154-139), dans Actas del Vil Congreso Español de Estudios Clásicos (Madrid, 1987), Madrid, 1989, pp. 257-262. On ne trouve cependant aucune mention des Vettons dans les textes, au moment de l'épisode de Viriathe, mais une traditionelle communauté d'intérêts avec les Lusitaniens, la localisation même de la Vettonnie entre le Mons Herminius et la Citerior (envahie par Viriathe) et le fait qu'en -139, Servilius, depuis ГUlterior, remonte vers le nord à la poursuite des Lusitaniens, dévastant les pays vetton et callaïque nous incitent à penser que les Vettons ont dû suivre la voie de la révolte généralisée. Il faut également penser à l'usage générique du terme de Lusitaniens.

Conimbriga, 40 (2001) 5-35 


\section{Traduction:}

Sous le consulat de Caius Marius et de Caius Flavius. Le peuple des Seano... se soumet à L. Caesius, fils de Caius, imperator. L. Caesius, imperator, après avoir reçu leur soumission, demanda au conseil ce qu'il estimait devoir leur demander. D'après l'avis du conseil, il demanda les captifs, les chevaux et les juments qu'ils auraient pris. Ils remirent tout. Après, L. Caesius, fils de Caius, ordonna que les terres et les bâtiments, les lois et toutes les autres choses qu'ils auraient eu jusqu'à leur soumission leur restent. Ils les leur rendit tant que le peuple et le sénat le voudraient. Sur ce, il ordonna aux légats d'aller... Creno et Arco, fils de Cantono, agirent en tant que légats ${ }^{8}$.

On peut interpréter cette tabula Alcantarensis, datée de 104 av. J.C., comme un traité de paix imposé par les Romains à une communauté (le populus Seano...), dont on n'a aucune trace par ailleurs, qui reprend les armes (ou continue de résister) à l'extrême fin du Ilème siècle, avant d'être vaincue'. Mais le fait que les Romains exigent de ce populus la restitution des captifs (captivos), des chevaux \{equos) et des juments \{equas), peut également traduire la fin d'une activité de banditisme (qui aurait subsisté dans certaines régions de manière endémique). En tout cas, le fait que le contenu du texte épigraphique (sur bronze) soit apparemment d'une relative modération (aucune destruction, aucune prise d'otages, aucune atteinte aux lois du populus $\mathrm{n}$ est exigée des Romains) semble indiquer que le problème devait être relativement minime, ne nécessitant pas de sanction exemplaire. Au con-

8 AE, 1984, 495; R. López Melero et alii, El bronce de Alcántara. Una deditio del 104 a.C., Gerión, II, 1984, pp.288-295; D. NòRR, Aspekte des rõmisches võlkerrechts. Die bronzetafel von Alcántara, Munich, 1989; L.A. García Moreno, Reflexiones de un historiador sobre el bronce de Alcántara, dans Epigrafia jurídica romana. Actas del coloquio internacional AIEGL (Pamplona, 1987), Pampelune, 1989, pp.243-255; S. MARINER BIgoRRA, La Tabula Alcantarensis entre la epigrafía jurídica arcaica de Hispania, dans ibidem, $\rho .257-263$; B.D. Hoyos, The dediticii of the Tabula Alcantarensis, ZPE, LXXVIII, 1989, pp.40-44; M.J. PENA, Importance et rôle de la terre dans la première période de la présence romaine dans la péninsule ibérique, dans Structures rurales et sociétés antiques. Actes du colloque de Corfou (1992), Paris, 1994, p.331.

9 II y eut d'autres problèmes avec les Lusitaniens (alliés aux Vettons?) dans les années 114-104: Plutarque, C. Gracchus, LXIV; ApPien, Iber., XCIX; Cicéron, Verr., IV, 56; Valere MAXIME, VI, 9, 13; sur cette communauté indigène, on pourra lire B.D. Hoyos, Populus Seanoc[...], 104 B.C., ZPE, LXXXIII, 1990, pp.89-95. 
traire, cette deditio doit être interprétée comme une mesure d'apaisement dans un contexte d'après-guerre où le souvenir des conflits viriathiques devait encore hanter l'esprit des proconsuls romains. C'est également le premier acte d'administration attesté à l'encontre d'une communauté lusitanienne ou vettonne dont le territoire devait alors relever de la provincia Ulterior ou bien n'était pas encore intégrée à cette dernière, précisément du fait de ces problèmes d'insécurité.

En effet, il nous faut rappeler ici quelques points importants à propos de l'intégration des Vettons dans les structures provinciales romaines qui se mettent en place:

- Nous ignorons la date précise à laquelle les Vettons ont été intégrés à $Y$ Ulterior: ce ne fut pas en tout cas, avant $-132^{10}$.

- Il est probable qu'une partie des Vettons aient relevé de la provincia Citerior, c'est ce que semblerait suggérer un texte de Pline qui situe, encore au ler siècle de notre ère, des Vettons dans les deux provinces: Citérieure et Lusitanie (mais ne s'agit-il pas d'une erreur de l'écrivain?) et un autre texte, de Plutarque qui nous apprend qu'en 95-94 av. J.C., le gouverneur de Citerior exigea des habitants de Bletisa (à l'est de Salmantica, sur le Tormes) qu'ils abandonnent leur coutume de sacrifier des êtres humains ${ }^{11}$. Pour ce dernier texte, on peut arguer que la Bletisa vettonne se situe dans une zone frontalière (entre Vettons et Vaccéens, à la marge de la Lusitanie et de la Citerior); il n'est donc pas surprenant, qu'à une époque où les limites provinciales sont sans doute encore assez floues, avec certainement des secteurs non provincialisés, que le gouverneur d'Ulterior intervienne dans une zone qui sera ensuite intégrée à la Lusitanie.

10 En -132, à la suite de la défaite de Numance, une commission sénatoriale est envoyée dans la péninsule en vue d'administrer les terres des vaincus (Lusitaniens et Celtibères nous précisent les sources, et sans doute d'autres peuples secondaires, en particulier les Vettons précisément localisés entre les Lusitaniens et les Celtibères): APPIEN, Iber., XCIX; selon E. AlBERTINI, Les divisions administratives de l'Espagne romaine, Paris, 1923, p.20, pense que cette commission aurait procédé à la délimitation des deux nouvelles provinces romaines.

11 Pline", III, 19, IV, 112, 113, 116; M.R. HeRnANDo SobrinO, La integración del territorio oriental de los Vettones en el marco administrativo-provincial romano, HAnt, XIX, 1995, pp.77-93, revient sur cette question et doute que les Vettons aient jamais pu se trouver à la fois en Lusitanie et en CiteriorPlutarque, Quaest.Rom., LXXXIII.

Conimbriga, 40 (2001) 5-35 
- Cinquante ans après cet épisode, César, dans sa Guerre civile, évoque une "Espagne citérieure», une Espagne "ultérieure du col de Castulo jusqu'à l'Anas»et le "pays des Vettones, à partir de l'Anas et la Lusitanie», chacune de ces «zones» étant dirigée par un légat de Pompée ${ }^{12}$. Il existait alors une grande province d' Ulterior (de l'océan au Guadiana) mais le territoire lusitano-vetton («àpartir de l'Anas et la Vettonnie») ne devait y être rattaché que depuis peu, peut-être du fait de son peuplement très différent de celui de 1' Ulterior méridionale (au sud du Guadiana) et de son caractère beaucoup moins ouvert sur le monde méditerranéen 13. En tout cas, nous avons là une préfiguration de ce que sera, quelques années plus tard, la réorganisation augustéenne de la péninsule, avec la création, à la place de $V$ Ulterior, des provinces de Lusitanie et de Bétique.

Auguste est en effet à l'origine de la division de VUlterior en deux provinces (Lusitanie et Bétique), ceci à partir de $-27^{14}$. Des motivations stratégiques expliqueraient, selon certains, la naissance d'une Lusitanie à vocation militaire, tournée vers le Nord-Ouest en partie insoumis. Cependant, force est de constater que la Vettonnie incluse en Lusitanie, n'a jamais été fortement militarisée malgré son voisinage avec les Astures ${ }^{15}$. Une nouvelle division se serait achevée au moment $\mathrm{du}$ voyage d'Auguste dans la péninsule, en 16-13 av.J.C ${ }^{16}$. On pensait que les territoires désormais soumis du Nord-Ouest avaient été alors

12 CAesar, Bellum civile, I, 38, 1; J.M. Roldán HevÂs, Fuentes antiguas para el estudio de los Vettones, Zephyrus, XIX-XX, 1968-1969, pp.95-96; P. LE Roux, Romains d'Espagne. Cités et politique dans les provinces lie siècle av. J.C.-IIIe siècle ap. J.C., Paris, 1995, pp.33-34.

13 JJ. Sayas Abengochea, Colonización y municipalización bajo César y Augusto: Bética y Lusitania, dans Aspectos de la colonización y municipalización de Hispania, Mérida, 1989, pp.107-134.

14 Dion Cassius, LUI, 12,4; Florus, II, 33,48; J.J. Sayas Abengochea, Algunas consideraciones sobre el origen de Lusitania como provincia, dans Estúdios dedicados a C. Callejo Serrano, Cáceres, 1979, pp.744-745.

15 La Vettonnie, à la lumière des sources épigraphiques, apparaît comme un cadre de recrutement (existence d'une ala Vettonum civium Romanorum aux Ier-IIème siècles) mais son caractère militarisé est peu affirmé: aucune colonie, aucune garnison n'y est attestée; l'archéologie révèle peu de fondations défensives et l'épigraphie ne nous fournit qu'une quinzaine de témoignages de militaires (légionnaires et auxiliaires) décédés en Vettonnie.

16 P. LE Roux, L'armée romaine et l'organisation des provinces ibériques, d'Auguste à l'invasion de 409, Paris, 1982, pp.54-84, 75, 306; R. ETIEnNE, L'horloge

Conimbriga, 40 (2001) 5-35 
rattachés à la Tarraconaise mais une récente découverte épigraphique atteste l'existence d'une provinda transduriana ${ }^{17}$. Mais cela n'enlève rien à la valeur de l'affirmation de Pline, IV, 113: «A Durius Lusitania indpit». Et c'est désormais dans ce nouveau cadre provincial, plus précisément dans sa partie nord et orientale, que la Vettonnie s'insère.

\section{La délimitation ( $c f$. Figure I)}

Nous avons déjà été amenés à nous intéresser à la délimitation de la Vettonnie sous le Haut-Empire, en relation avec les frontières de la toute nouvelle province de Lusitanie ${ }^{18}$. Cet aspect de l'intégration administrative des Vettons étant essentiel, il nous semble important de rappeler quelques données importantes:

Malgré la difficulté à déterminer une assise territoriale précise des Vettons, on notera, suite à nos propositions, qu'il y avait une relative concordance entre la limite ethnique des Vettons et les frontières provinciales, peut-être en raison de modifications frontalières intervenues à l'époque de Vespasien, en particulier avec le rattachement de Ladmur ga (à l'extrême sud de la Vettonnie) et d'Avila (est vetton) à la province de Lusitanie $^{19}$. Ainsi, après les modifications intervenues entre Pline et Ptolémée, on ne retrouve plus trace de Vettons ni en Bétique ni en Tarraconaise.

De plus, même s'il apparaît risqué de considérer les sculptures zoomorphes comme des critères très fiables pour localiser les Vettons, on constate que les verracos les plus septentrionaux, les plus orientaux

de la Civitas Igaeditanorum et la création de la province de Lusitanie, REA, XCIV, 1992, 3-4, pp.361-362, qui retient la date de -16 pour la réforme d'Auguste.

17 A. Rodríguez COLMENERO, El más antiguo documento (año 15 a.C.) hallado en el noroeste peninsular ibérico, CEG, XLVII, 112,2000, pp.9-41 (il s'agit d'un règlement entre des peuples gravé sur une plaque en bronze retrouvée dans le Bierzo).

18 C. Bonnaud, Vettonia Antiqua: les limites ethniques et administratives d'un peuple de l'ouest de la Meseta dans l'Antiquité (article sous presse).

19 J. MANGAS, La municipalización flavia en Hispania, dans Aspectos de la colonización y municipalización de Hispania, Mérida, 1989, pp.153-172; P. GUICHARD, Les effets des mesures flaviennes sur la hiérarchie existant entre les cités de la Péninsule ibérique, dans Ciudad y comunidad civica en Hispania (siglos II y III d.C.). Cité et communauté civique en Hispania (Madrid, 1990), Madrid, 1993, pp.67-84.

Conimbriga, 40 (2001) 5-35 
et les plus méridionaux de la Vettonnie sont localisés à proximité immédiate de la frontière provinciale ${ }^{20}$.

Il apparaît donc, et malgré les incertitudes pesant sur les verracos, que la limite entre la Lusitanie et les provinces de Tarraconaise et de Bétique se soient plus ou moins superposée aux frontières nord, est et sud de la Vettonnie. De même, l'unité vettonne n'a pas été entamée par la création du conventus de Mérida, et ce, contrairement aux Lusitaniens $^{21}$. Il y a bien eu, de la part des autorités romaines, la volonté de prendre en considération l'unité ethnique des Vettons. Cette reconnaissance de la réalité vettonne peut s'expliquer par un facteur essentiel: la localisation stratégique de leur territoire, entre Bétique et Nord-Ouest, le long d'un axe de circulation vital pour les Romains, à proximité immédiate de la capitale provinciale.

\section{Une provincia Lusitaniae et Vettoniae}

Un certain nombre de documents épigraphiques nous incitent à penser que l'intégration administrative des Vettons a été encore plus complexe ou a pu connaître des évolutions importantes. Il s'agit de cinq inscriptions dont aucune ne provient de Vettonnie, deux ont été retrouvées à Mérida, deux dans la province de Bétique et une, la plus endommagée, dans les eaux du Tibre 22 .

20 Voir figure I: les cadres ethnique et administratif et notre article, C. BONNAUD, Vettonia Antiqua: les limites ethniques et administratives d'un peuple de l'ouest de la Meseta dans l'Antiquité (sous presse), en particulier le paragraphe intitulé Peuplement vetton et limites de la Lusitanie: l'apport des verracos.

21 J. DE ALARCÀO et alii, Propositions pour un nouveau tracé des limites anciennes de la Lusitanie romaine, dans Les villes de la Lusitanie romaine. Hiérarchies et territoires. Table Ronde internationale $d u$ CNRS (Talence, 1988), Paris, 1990, pp. 325-327.

22 J.M. RoldÁn, Fuentes antiguas para el estudio de los Vettones, Zephyrus, XIX-XX, 1968-69, pp.79-80 et 98-100.

Conimbriga, 40 (2001) 5-35 


\begin{tabular}{l|l|l|l}
\hline PROVENANCE & NOM & TITULATURE & BIBLIOGRAPHIE \\
\hline Mérida & $\begin{array}{l}\text { C.Titius C.f. Cl(audia } \\
\text { tribu? ) Similis }\end{array}$ & $\begin{array}{l}\text { Procurator provinciae } \\
\text { Lusitaniae et Vettoniae }\end{array}$ & CIL II, 484 \\
\hline $\begin{array}{l}\text { Séville et Villalba } \\
\text { del Alcor (Huelva) }\end{array}$ & $\begin{array}{l}\text { M. Calpurnius M.f. } \\
\text { Gal. Seneca Fabius } \\
\text { Turpio Sentinatianus }\end{array}$ & $\begin{array}{l}\text { Procurator provinciae } \\
\text { Lusitaniae et Vettoniae }\end{array}$ & CIL II, 1178; CIL II, 1267 \\
\hline Rome & $\begin{array}{l}\text { L. Iulius Ve[hi?]lius } \\
\text { Gr[atus?] Iulianus }\end{array}$ & $\begin{array}{l}\text { Procurator provinciae } \\
\text { Lusitaniae et Vettoniae }\end{array}$ & CIL VI, 31856 \\
\hline Mérida & P. Aelius Vitalis & $\begin{array}{l}\text { Tabularius provinciae } \\
\text { Lusitaniae et Vettoniae }\end{array}$ & CIL II, 485 \\
\hline
\end{tabular}

Sur le plan chronologique, l'inscription retrouvée à Séville a été datée par H.G. Pflaum de l'année 130 apJ.C.23. Celle de $P$ Aelius Vitalis pourrait remonter au Ilème siècle apJ.C. à en juger par la formule D.M.S. Enfin, si l'on en croit J.M. Roldán, la première inscription de Mérida serait antérieure à Septime Sévère ${ }^{24}$. Il apparaît donc qu'il faille rejeter l'hypothèse selon laquelle la «province de Lusitanie et Vettonnie» aurait vu le jour dans le contexte troublé de l'Antiquité tardive et aurait été une réponse des autorités à un grand bouleversement ayant touché la province de Lusitanie.

En fait, l'existence d'une charge de procurator provinciae Lusitaniae et Vettoniae et d'une fonction de tabularius Lusitaniae et Vettoniae est surprenante dans la mesure où il n'existe pas d'autres documents (épigraphiques ou autres) mentionnant des fonctionnaires attachés à l'administration de la «province de Lusitanie et Vettonnie»"25.

L'interprétation d'une telle dénomination pose problème. Rappelons que le legatus augusti pro praetore exerçant la charge de gou-

23 H.G. Pflaum, Les carrièresprocuratoriennes équestres sous le Haut-Empire romain, II, Paris, 1960, pp.257-258, n. ${ }^{\circ} 107$.

24 J.M. ROLDÁN, art.cit., p.79.

25 A. BALIL, Los legados de Lusitania, Conimbriga, IV, 1965, pp.43ss; id., Funcionarios subalternos en Hispania durante el Alto Imperio, Emérita, XXXIII, pp.297ss; G.AlFõLdy, Fasti Hispanienses. Senatorische Reichsbeamte und Offiziere in den Spanischen Provinzen des Rõmisches Reiches von Augustus bis Diokletian, Wiesbaden, 1969; J.de Francisco MARTín, Conquista y romanización de Lusitania, Salamanque, 1989, pp.99-108.

Conimbriga, 40 (2001) 5-35 
verneur à Emerita était aidé pour les questions financières d'un procurator Lusitaniae de rang équestre. De ce dernier dépendaient d'autres procurateurs chargés de districts plus restreints. Des fonctionnaires subordonnés, esclaves ou affranchis de l'Empereur (P. Aelius Vitalis est Augusti libertus) avaient des tâches relativement précises, tels les tabularii qui devaient remplir des fonctions de comptables ${ }^{26}$.

Que ces procuratores et ce tabularius provinciae Lusitaniae et Vettoniae aient exercé des responsabilités financières en Lusitanie, cela ne fait pas le moindre doute, mais pourquoi la précision et Vettoniae? S'agit-il d'une mention marginale sans conséquence ou cela traduit-il une réalité administrative? E. Albertini pense qu'il s'agit là de la marque du rattachement à la Lusitanie des Vettons laissés dans un premier temps en Citerior ${ }^{27}$. On peut également avancer l'hypothèse de l'existence d'un régime fiscal particulier de la Vettonnie. Le fait que ces procuratores et ce tabularius aient été d'origine vettonne ou liés d'une quelconque façon à la Vettonnie (mariage, amitiés, clientèle, affaires) n'est pas impossible mais relève de la pure conjecture. Plus simplement n'est-ce pas là le témoignage de la volonté des autorités provinciales de prendre en considération la réalité vettonne en précisant que les Vettons, au même titre que les Lusitaniens, font partie de la province de Lusitanie. Il y aurait ainsi eu des périodes dans l'histoire administrative de la province où les Vettons auraient été l'objet d'une attention particulière. Le contexte précis d'un tel traitement nous échappe.

\section{LES STRUCTURES ADMINISTRATIVES LOCALES}

Comme l'a montré M. Salinas, la Lusitanie orientale, et tout particulièrement le pays vetton, présente une vie urbaine plus faible qu'ailleurs ${ }^{28}$. Cette faiblesse est compensée par d'autres formes de peuple-

26 J. Mangas et J.M. Solana SÁInZ, Historia de Castilla y León, II, Romanización y Germanización de la Meseta norte, Valladolid, 1985, pp.37-38.

27 E. AlBERTINI, op.cit., pp.115-116.

28 M. SALINAS, Las ciudades romanas de Lusitania oriental: su papel en la transformación del territorio y la sociedad indígena, dans Les villes de Lusitanie romaine. Hiérarchie et territoires. Table ronde internationale du CNRS (Talence, 1988), Paris, 1990, pp.255-263.

Conimbriga, 40 (2001) 5-35 
ment tels que les castros et les poblados, ces derniers constituant des noyaux non fortifiés. Cette faiblesse du réseau urbain, phénomène souvent organisateur d'un territoire environnant et donc générateur d'institutions locales, explique sans doute la difficulté qu'il y a à distinguer la réalité administrative et juridique de tous ces noyaux de peuplement. Les sources littéraires elles-mêmes sont d'une discrétion qui nuit gravement à notre approche de la question.

\section{Les lacunes des sources littéraires}

Strabon ne nous est guère utile dans sa Géographie car sa description est avant tout ethnographique. De plus, il n'est vraisemblablement jamais allé dans la péninsule ibérique d'où il aurait pu rapporter des informations intéressantes. Ainsi, ses mentions des Vettons ne comportent aucune précision administrative ou juridique. Pour Strabon, les Vettons constituent un «peuple», au même titre que les Lusitaniens, les Astures, les Vaccéens...De structures locales à l'intérieur de l'entité vettonne, Strabon n'en fait nullement mention ${ }^{29}$.

En revanche, on trouve dans les écrits de Pline quelques rares informations. Après avoir localisé des Vettons en Tarraconaise, celui-ci, dans le livre IV de son Histoire Naturelle, s'attarde sur la Lusitanie, qui commence à partir du Duero ${ }^{30}$. Les «gentes» de Lusitanie sont d'après Pline, les Celtici, les Turduli, les Vettones ("près du Tage») et les Lusitani. On remarquera cette utilisation du terme de gens pour traduire une réalité ethnique. Dans son étude sur les structures sociales, M.C. González Rodríguez a constaté que Pline, bien que procurator de Citerior, utilise à tort le terme de gens en lui conférant une signification très large: groupe ethnique (comme dans ce passage), population, habitants d'une ville, d'une région... ${ }^{31}$. Dans un autre passage, Pline, après avoir

29 P. PedeCh, La géographie urbaine chez Strabon, AncSoc, II, 1971, pp. 234-253; E.C.L. VAN DER VLIET, Strabon over landen, volken en steden, Assen-Amsterdam, 1977; A J. Domínguez MonEDERO, Reflexiones acerca de la sociedad hispana reflejada en la «Geografia» de Estrabón, Lucentum, III, 1984, p. 213; L. PÉREZ VILATELA, Etnias y divisiones interprovinciales hispano-romanas en Estrabón, Kalathos, IX-X, 1989-90, pp.205-214.

30 PLINE, III, 119; IV, 113, 116;

31 M.C. GONZÁLEZ RodRÍGUEZ, Las unidades organizativas indígenas del área indoeuropea de Hispania, Vitoria, 1986, pp.87-90.

Conimbriga, 40 (2001) 5-35 
rappelé l'organisation conventuelle de la Lusitanie, cite le chiffre de 45 populi, unités territoriales et administratives soumises à Rome, dont 5 colonies, un municipe de droit romain, trois municipes de droit latin et 37 cités stipendiâmes ${ }^{32}$. Parmi ces dernières, on peut considérer comme vettonnes, parce qu'elles sont connues par d'autres sources, les cités suivantes: les Augustobrigenses, les Caperenses, les Lancienses, les Ocelenses et les Caesarobrigenses. On notera que les Caurienses, cités par Pline, ne sont pas considérés comme vettons par Ptolémée ${ }^{33}$. Ce passage de Pline mérite quelques remarques. En effet, Pline ne mentionne de communautés vettonnes que dans le groupe des cités stipendiâmes, c'est-à-dire non privilégiées sur le plan juridique. Or, Pline, surtout en tant que procurateur, est censé maîtriser les différences entre colonies, municipes et cités stipendiâmes. Ainsi, le fait qu'il ne mentionne aucune colonie vettonne nous incite à penser qu'il sait de quoi il parle $^{34}$. A priori donc, la formule de Pline est acceptable et refléterait la situation administrative d'une époque relativement précise. Mais une autre constatation s'impose: toutes les communautés vettonnes ne sont pas citées par Pline: Salmantica, Bletisa, Avela, Lacimurga, pour ne mentionner que celles qui ont été localisées, sont absentes de sa liste.

32 Pline, IV, 118; B. Galsterer-Kroll, Zu den spanischen Stàdte des Plinius, AEA, XLVIII, 131-132, 1975, pp.120-128.

33 M. SALINAS, La organización tribal de los Vettones (Pueblos prerromanos de Salamanca), Salamanque, 1986, p.39, incluerait Coria en Vettonnie malgré Ptolémée, II, 5,6; J.M. FERnÁNDEZ CORRALES, El asentamiento romano en Extremadura y su anális espacial, Cáceres, 1988, p.36, pense que Pline s'est trompé en affirmant que Coria est lusitanienne, ce qui équivaut à dire qu'elle est vettonne. En fait, cette ambiguïté ethnique est liée sans doute à la situation frontalière de la civitas Cauriensis, entre la civitas Caper ensis (vettonne) et la civitas Igaeditanorum (lusitanienne). Nous nous situons là dans une zone de passage, sur la rive d'un affluent important et sans doute très fréquenté du Tage: l'Alagón. Coria était également desservie par une voie romaine reliant Alconétar à Ciudad Rodrigo. Il pouvait s'agir d'une ville anciennement vettonne, ayant subi la pression lusitanienne, dans une zone où les deux peuplements devaient s'entremêler. Durant des siècles, Coria a eu un rôle de ville frontière, à quelques kilomètres du Portugal.

34 L. BracCESI, Plinio Storico. Plinio il Vecchio sotto il profilo storico e letterario, dans Atti del Convegno di Como (1979), Corne, 1982, pp.53-82; A. GuERRA, Plinio-o-Velho e a Lusitania, Lisbonne, 1995; G. FÁTAS, La polis indígena. Notas metodológicas, dans Estudios de Historia de España. Homenaje a M.Tuñon de Lara, Madrid, 1981, évoque cependant le manque d'exactitude des termes de populus et de civitas dans le livre III de la Naturalis Historia.

Conimbriga, 40 (2001) 5-35 
Et il est difficile de croire que ces noyaux, en particulier Salmantica, avaient un statut juridique inférieur (des à Lancia ou Ocelum par exemple, ce qui expliquerait que Pline ne les ait pas mentionnés. De plus, si on peut à la rigueur justifier l'absence d ou de Lacimurga, qui appartenaient alors l'une à la Tarraconaise et l'autre à la Bétique, Salmantica et Bletisa n'ont jamais appartenu à une autre province que la Lusitanie.

La liste des villes vettonnes de Ptolémée a l'avantage de bien faire ressortir ces oublis de Pline. Outre les quatre «villes» - terme utilisé par Ptolémée - citées précédemment et que l'on ne trouve pas dans le texte de Pline, le géographe grec mentionne quatre autres noyaux urbains que Pline a également passés sous silence: Koxxarôppiya, MavAxava, Asôpptya et $\mathrm{Aapa}^{35}$. Ces villes devaient avoir un statut juridique à l'époque de Pline, à moins qu'elles n'aient toutes été des créations postérieures, ce qui est peu probable. De plus, dans le terme de JioAaç, Ptolémée inclut peut-être des noyaux très secondaires.

\title{
Epigraphie et promotion municipale
}

\author{
MVNICIPIA \\ PROVINCIAE \\ LU SITANIAE.STIPE \\ CONLATA.QVAE.OPVS \\ PONTIS.PERFECERVNT \\ IGAEDITANI \\ LANCIENSES.OPPIDANI \\ TALORI \\ INTERANIENSES \\ COLARNI \\ LANCIENSES.TRANSCVDANI \\ ARAVI \\ ME ID VBRIGENSES \\ ARABRIGENSES \\ BANIENSES \\ PAESVRES ${ }^{36}$
}

35 PTOLEMEE, II, 5, 7.

36 CIL II, 760; H. GALSTERER, Untersuchungen zum romischen Stadtewesen aufder iberische Halbinsel (MF, VIII), Berlin, 1971, pp.62-64; L. GARCÍA IGLESIAS,

Conimbriga, 40 (2001) 5-35 
Cette liste des municipes de la province de Lusitanie ayant participé, sous Trajan, à la rénovation du pont d'Alcántara, est intéressante à divers titres. De cette liste, extrayons d'abord ce qui nous apparaît comme vetton: les Lancienses Oppidani et, sans doute, les Lancienses Transcudani $^{37}$. Les autres peuples mentionnés sont lusitaniens ${ }^{38}$. Il est intéressant de voir que non seulement il n'y a plus une (Pline, IV, 118, Ptolémée, II, 5, 7) mais deux Lancia, toutes les deux vettonnes si on retient l'hypothèse d'un dédoublement de l'oppidum, mais encore, il n'y a plus de populus stipendiaire (Pline), mais deux municipiae stipendiâmes, comme d'ailleurs le reste des peuples de l'inscription qui auraient tous reçu, à cette occasion, leur promotion municipale ${ }^{39}$. La question est de savoir si cette promotion municipale est liée à la réparation du pont et donc limitée, ou si l'ensemble des communautés vettonnes a connu cette promotion, et à quelle époque.

En fait, le cas de Lancia ne semble pas isolé. Pline considère $\mathrm{Ca}$ pera, aujourd'hui parfaitement localisée (nord de la province de Cáceres), comme un simple populus stipendiarius ${ }^{40}$. Or, comme nous le verrons, il semble bien qu'au début du Illème siècle ap.J.C., Capera soit un réel municipe, d'après un document épigraphique mentionnant un magistrat de cette ville. Selon M. Salinas, cette municipalisation a pu

Autenticidad de la inscripción de los municipios que sufragaron el puente de Alcántara, REE, XXXII, 1976, pp.262-275; B.D. HoYOS, In defence of CIL II, 760, Athenaeum, LXVI, 1978, pp.390-395; A. TRANOY, L'organisation urbaine dans le conventus scallabitanus, dans Les villes de la Lusitanie romaine. Hiérarchies et territoires. Table ronde internationale du CNRS (Talence, 1988), Paris, 1990, pp. 18-20.

37 J.M. RoLdÁn, Fuentes antiguas para el estudio de los Vettones, Zephyrus, XIX-XX, 1968-69, pp.73-106; M. SALINAS, Las ciudades romanas de Lusitania oriental: su papel en la transformación del territorio y la sociedad indígena, dans Les villes de Lusitanie romaine. Hiérarchie et territoires. Table ronde internationale du CNRS (Talence, 1988), Paris, 1990, p.258; sur la localisation des deux Lancia, cf. J.de ALARCÀo, Roman Portugal, I, Introduction, Warminster, 1988, pp.18-19.

38 R. HuRTADO DE SAN ANTONIO, Identificación de los municipia estipendiarios que sufragaron el puente romano de Alcántara, REE, XXXII, 2, 1976, 605-622; J. de ALARCÃO, Identificação das cidadas da Lusitânia portuguesa e dos seus territórios, dans Les villes de Lusitanie romaine. Hiérarchies et territoires. Table Ronde Internationale du CNRS (Talence, 1988), Paris, 1990, pp.27-34.

39 J.L. SAyas AbengocheA, Municipalización de la Hispania romana. Ideología y realidad, dans Centralismo y Decentralización. Modelos y procesos históricos en Lrancia y en España (Madrid, 1984), Madrid, 1985, pp.141-146.

40 Pline, IV, 118. 
intervenir dès la fin du 1er siècle ap. J. C.41. En effet, la mention de la tribu Quirina, sur un autre document, est à mettre en relation avec l'œuvre de municipalisation des Flaviens ${ }^{42}$. De plus, selon P. Le Roux, Caesarobriga (Talavera de la Reina, ouest tolédan) a également été concerné par ce mouvement. En revanche, Augustobriga (Talavera la Vieja, est de la province de Câceres) en aurait été exclue ${ }^{43}$.

Il nous semble utile de nous attarder sur les témoignages épigraphiques qui nous révèlent les traces concrètes de cette municipalisation, c'est-à-dire les institutions ${ }^{44}$. Capera est sans doute l'exemple le plus probant de cette municipalisation. Salmantica et Caesarobriga ont également connu cette promotion. Mais d'autres villes ont-elles été concernées?

\section{Capera}

Un des documents qui nous intéresse a d'abord consisté en trois «fragments d'une inscription gravée sur de beaux blocs de calcaire» ${ }^{45}$. Le mauvais état de la stèle explique les différentes tentatives de restitution du texte, tentatives qui demeurèrent peu convaincantes et ne parvinrent pas à une compréhension générale de l'inscription ${ }^{46}$. Mais la découverte postérieure d'un quatrième fragment a permis de reconstituer la plus grande partie de l'inscription, en dépit de deux blocs toujours manquants ${ }^{47}$ :

$$
\begin{aligned}
& \text { [Pro.sa]LVTE.MVNICIP(i).FLAV(i).CA[perens(is)] } \\
& \text { AQVA.AVGUSTA } \\
& {[\ldots . .] \text { ALBINVS.[ex].TE[st]AMENTO.[f(ieri) iussit?] }{ }^{48} \text {. }} \\
& 41 \text { M. SAunas, art.cit., p.238. } \\
& 42 \text { R. WIEGELS, Die Tribusinschriften des romischen Hispanien. Ein Katalog, Ber- }
\end{aligned}
$$
lin, 1985; R. KNox MAC EldeRRY, Vespasian's reconstruction of Spain VIII. 1918, pp.53ss; A. MontÉnÉGro, Problemas y nuevas perspectivas en el estudio de la Espana de Vespasiano, Hant, V, 1975, pp.7-88; J. Muniz Coello, La politica municipal de los Flavios en Hispania. El municipium Imitanum, SHHA, II-III, 1, 1984-85, pp.151-176.

43 P. LE Roux, Les villes de statut municipal en Lusitanie romaine, dans Les villes de Lusitanie romaine. Hiérarchies et territoires. Table ronde internationale $d u$ CNRS (Talence, 1988),Paris, 1990, p.45.

44 L A . CURChin, The local magistrates of Roman Spain, Toronto, 1990.

45 R. Thouvenot, Note sur trois inscriptions lusitaniennes, $R E$ A, XLII, 1940, p. 531.

${ }^{46} A E, 1941,133$.

47 J.M. Blázquez, Cáparra, II (EAE, LIV), Madrid, 1966, pp.36ss; HAE, 2584.

$48 A E, 1986,307$.

Conimbriga, 40 (2001) 5-35 
Nous reproduisons également ci-dessous la reconstitution de l'inscription telle qu'elle a été effectuée par A.U.Stylow:

\section{PRO.SALVTE.MVNICIPI.FLAVI. CAPERENS$$
\text { AQVAAVGVSTA }
$$$$
\text { — ALBINVS..EX.TESTAMENTO-_ }
$$

Nous avons ainsi la preuve qu'il existait un municipium Flavium Caperense auquel devait appartenir

Outre le caractère assez particulier de cette inscription, liée à l'importance de l'eau dans la cité, nous sommes sans doute en présence d'un texte d'époque flavienne voire trajane. A.U. Stylow déduit ces indications chronologiques de l'indication sans abréviation du statut privilégié de Capera, de la forme des lettres (en particulier la barre du $T$ de $S A L U T E$ ). L'utilisation de l'épithète Augusta n'aurait qu'une valeur générique. L'examen de ce document ne permet cependant pas de savoir à quel empereur flavien on doit l'élévation de la cité au rang de municipium Flavium iuris Latini.

Une autre inscription de Capera, dédiée à la divinité Trebaruna par M. Fidius Fidi f. Quir(ina) Macer, confirme le statut municipal de la cité:

$$
\begin{gathered}
\text { AVG(ustae) TREBAR(unae) } \\
\text { M(arcus) FIDIVS.FIDLF(ilius).QVIR(ina tribu) MACER } \\
\text { MAG(ister) IIIJLVIR.PRAEF(ectus).FA[brum }]^{50} .
\end{gathered}
$$

Ainsi, ce personnage, après avoir été magister (ou magistratus) trois fois dans sa cité (sans doute stipendiaire) semble être devenu duumvir, charge qu'il exerça à deux reprises, avant d'être praefectus fabrum. Ce membre de l'élite locale a dû connaître une carrière parallèle à l'évolution juridique de sa cité. Sa fonction de duumvir du nouveau municipe de Capera semble lui avoir ouvert les portes de l'ordre équestre puisqu'il fut également Préfet des Ouvriers ${ }^{51}$. Certains ont

49 P. LE Roux, art.cit., p.45; A.U. STYLow, Apuntes sobre epigrafia de época flavia, Gerión, IV, 1986, pp.306-307.

$50 A E, 1967,197=A E, 1987,616$ j; H. Galsterer, op.cit., p.68 n. ${ }^{\circ}$ 5; J. de Francisco MARTIN, Los magistrados municipales en Lusitania durante el Alto Imperio, MHA;I, 1977, p.235 n. ${ }^{\circ} 24$.

51 E. GIL GarcîA, Los praefecti fabrum en la Península Ibérica, dans II Congresso peninsular de História Antiga (Coimbra, 1990), Coïmbre 1993, pp.753-765.

Conimbriga, 40 (2001) 5-35 
évoqué l'absence de toute mention d'une carrière sacerdotale ${ }^{52}$.On peut préciser qu'il ne s'agit pas d'une stèle funéraire et que Fidius Macer a pu revêtir par la suite un honneur religieux. La mention de la tribu Quirina, rappelons-le, peut être mise en relation avec l'œuvre de municipalisation des Flaviens et justifie la datation de J.M. Blâzquez: la fin du 1er siècle ap J.C. ${ }^{53}$.

Une dernière inscription de Capera, plus tardive, est un autre témoignage de la promotion juridique de la cité:

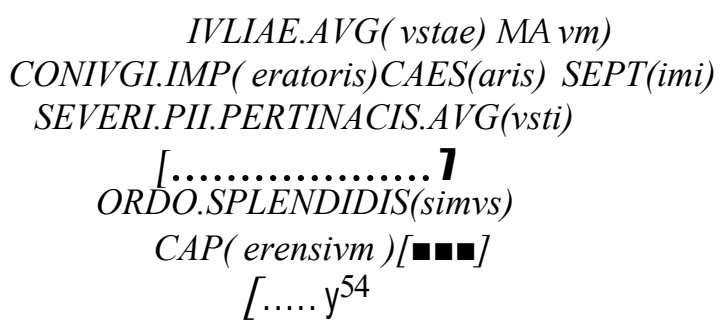

Sur le plan strictement administratif, on remarquera l'existence, au début du Illème siècle, puisqu'il s'agit d'une dédicace à Septime Sévère et à son épouse Julia Domna, d'un ordo splendidissimus Caperensium qu'on pourrait fort bien mettre en relation avec le statut municipal de la ville 55 .

\section{Salmantica}

Malgré l'absence de cette ville dans le texte de Pline, et en dépit du témoignage de Frontin qui n'y voit qu'un vicus, Salmantica, en raison de sa localisation idéale sur le Tormes, sur la voie romaine reliant Mérida à Astorga, à la limite de la Vettonnie et du pays vaccéen, en Lusitanie mais non loin de la Tarraconaise, ne pouvait être une simple bourgade comme le suggère $\mathrm{V}$. Bejarano ${ }^{56}$. La promotion municipale

52 R. ETIENNE et F. MAYET, DU nouveau sur Capera-Capara, REA, LXXIII, 1971, p.387.

53 J.M. BLÂZQUEZ, Cáparra, I (EAE, XXXIV), Madrid, 1965, p.59.

54 CIL /7,810

55 P. LE Roux, art.cit., p.45.

56 V. BEJARANO, Fuentes antiguas para la historia de Salamanca, Zephyrus, VI, 1955, pilló. 
de la ville a peut-être été plus tardive qu'ailleurs en Vettonnie (il ne s'agit pas d'une création romaine mais d'une ville indigène) mais elle a cependant eu lieu, comme semble l'attester un document épigraphique (gravé sur piédestal), conservé au Musée provincial de Salamanque. Il s'agit d'une dédicace à l'empereur $M$. Antoninus Caracalla fait par un ordo salmantic(.. . $)^{57}$ ( $c f$. Figure II). Au début du Illème siècle, Salmantica aurait donc atteint le rang de municipe ${ }^{58}$. De plus, on pourrait, à la suite de $\mathrm{M}$. Salinas, invoquer, en faveur du statut municipal de Salamanque, les récentes découvertes archéologiques effectuées dans le sous-sol de la Bibliothèque de Physiques de l'Université: on a pu identifier un quartier aisé avec un aqueduc, des demeures aux murs stuqués et peints de motifs géométriques, de la terra sigillata en abondance, ainsi que de la céramique de luxe à parois fines. De tels éléments se retrouvent à Chaves ou à Câparra après l'octroi du statut de municipe ${ }^{59}$.

\section{Caesarobriga}

L'hypothèse d'une création augustéenne n'est pas à écarter ${ }^{60}$. P. Le Roux considère Caesarobriga (Talavera de la Reina) comme un municipe flavien ou post-flavien ${ }^{61}$. L'épigraphie confirme-t-elle cette promotion juridique? Outre l'abondance du matériel épigraphique, ce qui ne prouve rien, et l'onomastique nettement plus romanisée qu'à Salamanque par exemple, nous avons la preuve de l'existence d'institu-

57 P. LE Roux, art.cit., p.46, ne retient pour Salmantica, comme pour Bletisa, Caetobriga et Mirobriga, que le rang d'oppidum Latinum.

58 J. de Francisco MARTín, Salamanca, municipio romano y la municipalización de Lusitania, dans II Congresso Peninsular de Historia Antiga (Coimbra, 1990), Coïmbre, 1993, p.623.

59 Communication personnelle.

60 G. AlFõLdy, Romisches Stüdtewesen aufder neukastilischen Hochebene. Ein Testfall fur die Romanisierung, Heidelberg, 1974, p.58; D. NON Y, Les provinces hispaniques, dans C. LEPELLEY, Rome et l'intégration de l'Empire, t.2, Approches régionales du Haut-Empire romain, Paris, 1998, pp.117 et 136 fait de Caesarobriga une ville neuve pour regrouper les indigènes; sur la politique urbaine de César et d'Auguste cf. J J. SAYAS ABENGOCHEA, Colonización y municipalización bajo César y Augusto: Bética y Lusitania, dans Aspectos de la colonización y municipalización de Hispania, Mérida, 1989, pp. 107-134.

61 P. LE Roux, art.cit., p.45. 
tions municipales avec un document mentionnant un certain L. Annius Placidus de la tribu Quirina, qui fut édile, questeur puis duumvir trois fois, avant de trouver la mort à 40 ans, mention intéressante dans la mesure ce document nous indique qu'on pouvait fort bien parvenir à de hautes fonctions municipales à un âge peu avancé:

\author{
D.M.S. \\ L. ANNIO PLACI \\ DO QU1R.CAESA \\ ROBRIG.ANXL \\ AEDI.QVAESTO \\ RI IIVIRO TER \\ DOMITIA ATTIA \\ MARITO OPTIMO ${ }^{62}$.
}

Une autre stèle évoque une Domitia Proculina, flaminique de Lusitanie et première flaminique perpétuelle de son municipe:

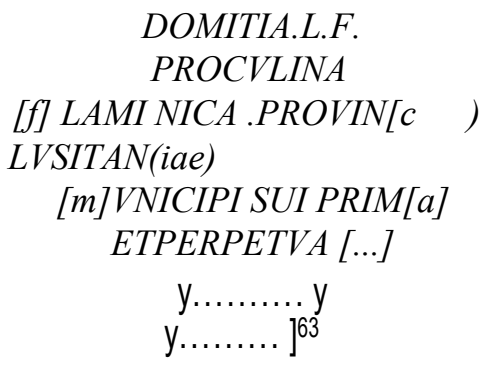

Selon J. de Francisco Martin, ces deux personnages, membres de l'élite municipale, pourraient être apparentés ${ }^{64}$. La datation flavienne de R. Etienne se justifierait par la mention flaminica municipi sui prima: une fois sa cité devenue municipe, Domitia Proculina «a eu à cœur de

62 CIL IL 896; le document a été retrouvé en 1757 dans les murailles de la ville de Talavera de la Reina, avant d'être incorporé à la fontaine des anciennes usines royales.

63 CIL $/ 7,895$.

64 J. de FRANCISCO MARTín, LOS magistratos municipales en Lusitania durante el Alto Imperio, $M H A$, I, 1977, p.234 n. ${ }^{\circ} 20$; on notera cependant que la stèle du magistrat a été érigée par son épouse Domitia Attia le fait que la flaminique s'appelle Proculina Domitia ne nous semble pas un critère suffisant pour attribuer un lien de parenté entre les deux femmes.

Conimbriga, 40 (2001) 5-35 
se dire la première» ${ }^{65}$. Ainsi, Caesarobriga non seulement était un municipe où s'exerçait le culte impérial depuis l'époque flavienne, mais encore était revêtue du privilège d'envoyer dans la capitale provinciale une flaminique (élue par le concilium). Pour essayer d'expliquer ce privilège accordé à cette ville vettonne on peut avancer l'idée d'un dynamisme du culte impéral à Caesarobriga, d'où un certain rayonnement de la cité par ailleurs très bien reliée à la capitale provinciale ${ }^{66}$.

\section{Mirobrigai}

Si la Mirobriga citée par Pline (IV, 118) n'est sans doute pas en Vettonnie mais dans le territoire des Celtici (conventus Pacensis), nous connaissons l'existence, par plusieurs termini augustales, d'une Mirobriga vettonne, dont le territoire a été l'objet de modifications à l'époque augustéenne ${ }^{67}$. Acceptant l'identification de cette Mirobriga à l'actuelle Ciudad Rodrigo (sud-ouest de la province de Salamanque), une dédicace à l'empereur Domitien pourrait être un argument en faveur de la municipalisation de cette ville:

$$
\begin{gathered}
\text { IMP(eratori) CAES(ari) DIVI } \\
\text { VESPASIANI F (ilio) DOMITIANO AVG(usto) } \\
\text { PONT(ifici) MAX(imo) TRIB(unicia) } \\
\text { P(otestate) IMP(eratori) II P(ater) P(atriae) CO(n)S(uli) } \\
\text { VIII DESIG(nato) VIIII } \\
\text { D(ecreto) D(ecurionum })^{68} .
\end{gathered}
$$

Cette dédicace à l'empereur vivant Domitien peut être datée de 82 ap J.C. Domitien fut salué imperator pour la seconde fois et obtint le

65 R. ETIENNE, Le culte impérial dans la péninsule ibérique d'Auguste à Dioclétien, Paris, 1974, p. 167.

66 R. ETIENNE, op.cit., p.219: «L'élection du flaminique provincial ou de la flaminique provinciale manifeste le poids de la cité à laquelle ils appartiennent».

67 CIL II, 857, 858, 859 et 5033.

68 CIL II, 862; C. MoRÁN, Epigrafia salmantina, Salamanque, 1922, p.56, n. ${ }^{\circ} 113$; R. MARTín VALlS, Nuevos hallazgos arqueológicos en Ciudad Rodrigo, Zephyrus, XXVI-XXVII, 1976, p.387; J. MANGAS, Ciudades antiguas de la Provincia de Salamanca (siglo II a.C; - Diocleciano), dans Primer Congreso de Historia de Salamanca, I, Salamanque, 1992, p.262. 
consulat pour la huitième fois cette année-là soit peu de temps après la mort de Titus ${ }^{69}$. L'autel semble avoir été érigé par les décurions de la ville (Decreto Decurionum) dans un contexte assez précis: celui d'un intérêt tout particulier porté à la péninsule ibérique par les Flaviens, en particulier par Vespasien qui procéda à une réorganisation administrative (création des conventus), accorda le ius latii aux Hispaniques, créa de nouveaux municipes \{MirobrigaT) et renouvela le culte impérial ${ }^{70}$. Mais il est possible que la promotion municipale de Ciudad Rodrigo date $\mathrm{du}$ règne de Domitien lui-même, d'où cette dédicace. En tout cas, l'absence de martelage sur ce document, comme conséquence de la damnatio memoriae de Domitien pourrait s'expliquer par un certain attachement à l'empereur, malgré ses excès, de la part du milieu dirigeant de cette ville ${ }^{71}$. Cependant, la promotion municipale de Mirobriga n'est pas du tout certaine car l'existence de décurions n'est pas caractéristique des municipes ${ }^{72}$.

\section{Vrunia?}

Une autre dédicace, cette fois à l'empereur Septime Sévère, a été retrouvée à Irueña (territoire de Fuenteguinaldo), à quelques kilomètres au sud-ouest de Ciudad Rodrigo:

$$
\begin{gathered}
\text { IMP( eratori).CAES( ari ). } \\
\text { L(ucio ) SEPTfimio ) SEVERO } \\
\text { PERTINACI AVG( usto ). } \\
\text { O(rdo ) M(unicipii).V( runensis ).EX. } \\
\text { A(rgenti).P( ondo ). } .{ }^{73}
\end{gathered}
$$

69 I. CALABI LAMENTAM, Epigrafia latina, Milan, 1974, p.481.

70 A.B. BoswORTH, Vespasian and the provinces: some problems of the early 70's A.D., Athenaeum, LXI, 1973, pp.49-78; P. GUICHARD, Les effets des mesures flaviennes sur la hiérarchie existant entre les cités de la Péninsule ibérique, dans Ciudad y comunidad cívica en Hispania (siglos II y III d. C.). Cité et communauté civique en Hispania (Madrid, 1990), Madrid, 1993, pp. 67-84; sur la mention D.D., cf. J. d'ENc ARN AÇÃO, Algumas notas sobre o mecanismo decisório municipal na Hispânia romana, dans Ciudad y comunidad cívica en Hispania (siglos II y III d.C.). Cité et communauté civique en Hispania (Madrid, 1990), Madrid, 1993, pp.59-64.

71 R. ETIENNE, op.cit., pp.457-458.
${ }_{72}$ P. LE Roux, art.cit., p.46.
73 CIL /7,863. 
En acceptant le texte de E. Hübner, nous serions en présence d'un municipe dont 1'ordo, sorte de sénat de la ville, aurait fait ériger un monument de cinq livres d'argent à Septime Sévère. Nous ne possédons malheureusement plus ce document qui semble douteux, bien qu'il devait exister un noyau urbain relativement important à Irueña, d'après les vagues descriptions de J. Maluquer et de J. Mangas ${ }^{74} 7576$ 77. En fait, la restitution des trois dernières lignes sont fortement suspectes, à commencer par la restitution $V$ (runensis). C. Morán, pour sa part, transcrit $V$ (alutensis) ${ }^{15} \mid$ il est vrai qu'une Valuta serait par ailleurs attestée au contact des cités de Mirobriga, Salmantica et Bletisa ${ }^{16}$. Il nous semble encore plus hasardeux de voir dans le $M$ une abréviation de Municipii, comme le pense J. Mangas qui lit $O($ rdo $)$. MIR(obrigensis $)^{11}$.

Ainsi, rien ne nous permet d'affirmer sans le moindre doute la promotion municipale de cités comme Mirobriga, Vrunia (?) ou Valuta (?). Une seule chose est certaine: des noyaux de peuplement vettons, et peut-être pas des moindres, demeurèrent de simples communautés indigènes non privilégiées sur le plan juridique. Ces cités pouvaient être à la tête de vastes territoires ou être parées d'un décor monumental imposant comme à Augustobriga, ville conçue à la romaine en bordure du Tage, possédant un senatus et un populus sans pour autant que tout cela soit la preuve d'une quelconque promotion juridique ${ }^{78}$. De même, Bletisa, l'actuelle Ledesma, sur le Tormes, et Polibeda, attestée sur une borne augustale retrouvée à Yecla de Yeltes (ouest salmantin) ne semblent jamais avoir eu d'institutions indiquant une promotion municipale $^{79}$. Enfin, dans le castro de Las Merchanas, toujours dans l'ouest de la province de Salamanque, un fragment de tessère d'hospitalité ne fait mention que de simples magistrati, à l'intérieur d'un petit noyau de peuplement (environ 5 hectares de superficie) ${ }^{80}$.

74 J. Maluquer, Carta arqueológica de España. Salamanca, Salamanque, 1956, p. 63 ss; J. MANGAS, art.cit., p.262.

75 C. MORAN, op.cit., p. 57 n. ${ }^{\circ} 114$.

76 CIL II, 857 et 858.

77 J. MANGAS, art.cit., p.263; R. MARTín VALLS, art.cit., p.388, affirme d'ailleurs que la dédicace en l'honneur de Septime Sévère provient en fait de Ciudad Rodrigo.

78 CIL II, 5346; P. LE RouX, art. cit., p.45; R. WIEGELS, Die Tribusinschriften des rômischen Hispanien. Ein Katalog (MF, XIII), Berlin, 1985, p.73.

79 J. MALUQUER, op. cit., p. 122.

80 J. MALUQUER, op.cit., p.74; J. MANGAS, art.cit., pp.264-265. 


\section{Dimensions chronologique et juridique}

Il semble utile, étant donné la complexité de la question municipale, de rappeler l'évolution et l'état actuel de la recherche concernant ce sujet, et en particulier le problème des effets des mesures flaviennes (droit latin accordé à toute l'Hispanie par Vespasien) sur les statuts et institutions des noyaux de peuplement ${ }^{81}$.

En 1965, Ch. Saumagne soutenait la thèse selon laquelle, dès l'époque de César ou d'Auguste, les municipes des provinces romaines étaient exclusivement des municipes de droit latin ${ }^{82}$. Les études postérieures de $\mathrm{H}$. Braunert et B. Galsterer-Kroll ont permis de noter l'absence de lien automatique entre l'octroi du droit latin par Vespasien et la promotion d'une communauté au rang municipal83. Malgré cette promotion, nous savons que la forme politique indigène, ce que les Romains appelaient la civitas, s'est conservée et que le statut latin était une catégorie juridique personnelle, sans influence sur la forme municipale, entraînant seulement des modifications pour certains individus: l'accession à la citoyenneté romaine par le biais d'un cursus honorum local (ius adipiscendae civitatis per magistratum). Les travaux de P. Le Roux ont permis une grande avancée dans la connaissance de ce phénomène ${ }^{84}$. Cet historien, sans s'attarder sur le cas de la Vettonnie, pense que la province de Lusitanie a connu une municipalisation active, en particulier dans le cadre de l'octroi du ius Latii à toutes les cités d'Espagne. Il conteste l'idée de $\mathrm{H}$. Galsterer selon laquelle le droit latin n'aurait pas été étendu aux régions les moins romanisées et les moins

81 J. MANGaS, La municipalización flavia en Hispania, dans Aspectos de la colonización y municipalización de Hispania, Mérida, 1989, pp. 153-172; P. GUICHARD, art.cit., pp. 67-84; P. LE RoUX, Romains d'Espagne. Cités et politique dans les provinces lié siècle av. J.C. - Illè siècle ap.J.C., Paris, 1995, p.83-87.

82 C. SAUMAGne, Le droit latin et les cités romaines sous l'Empire, Paris, 1965.

83 H. BRAUNERT, Ius Latii in den Stadtrechten von Salpensa und Malaca, dans Rômische Forschungen in Niederõsterreich, $V$, Corolla Memoriae Erich Swoboda dedicata, Graz-Cologne, 1966, pp.7Iss; B. GALsterer-Kroll, Zum Ius Latii in den Keltischen Provinzen des Imperium Romanum, Chiron, III, 1973, pp.277-306.

84 P. LE Roux, Municipe et droit latin en Hispania sous l'Empire, RHDFE, LXIV, 1986, pp.325-350; id., art. cit., pp.35-49; id., La questione municipale nel I secolo d.C.;: l'esempio spagnolo, dans Epigrafia e territorio. Politica e societá. Temi di Antichitá romane, III, Barí, 1994, pp.159-173; on pourra également lire A. CHASTAGNOL, A propos du droit latin provincial, lura, XXXVIII, 1987 (1990), pp.1-24. 
urbanisées de la péninsule ${ }^{85}$. Et nous avons vu que la Vettonnie, qu'il faut incontestablement ranger dans ce type de région, a été concernée par la municipalisation; celle-ci impliquerait l'existence préalable d'un oppidum Latinum à partir de Vespasien. Le droit latin facilitait l'intégration des élites provinciales dans la cité romaine et y facilitait la promotion municipale. Comme l'ont rappelé P. Le Roux et A. Tranoy, le statut municipal n'était pas la condition nécessaire d'une urbanisation selon les formes romaines ${ }^{86}$. L'exemple de la Vettonnie est à cet égard parlant: les probables municipes ne présentent aucun caractère d'urbanisme plus sophistiqué que les autres noyaux urbains. Le problème qui se pose est celui de la sélection, du choix des oppida Latina destinés à devenir des centre administratifs autonomes. S'appuyant sur l'exemple des tables en bronze du municipium Irnitanum, P. Le Roux a défini un certain nombre de critères pour accéder au rang municipal, au premier rang desquels l'acceptation des habitants, des conditions financières rendant possible la gestion locale d'une communauté, la certitude d'une administration locale de la justice...87. Par le biais de la municipalisation, une ville développait son emprise sur un espace environnant; une élite municipale accroissait son influence sur une population, sans doute peu nombreuse, répartie sur le territoire du municipe. A la lumière de ces informations, la situation et l'évolution juridico-administrative des communautés vettonnes nous apparaissent un peu plus clairement.

L'empreinte coloniale et municipale de l'époque césaro-augustéenne a peu marqué, semble-t-il, les régions vettonnes. En revanche, cela n'est pas incompatible avec un effort important dans l'organisation territoriale des civitates, sous le principat d'Auguste. Ainsi, l'épigraphie témoigne d'une importante activité de délimitation et de bornage des territoires de Lancia Oppidana, Polibeda, Mirobriga, Bletisa, Sal-

85 P. LE Roux, c.r. du Untersuchungen de H. Galsterer, REA, LXXIV, 1972, pp.410-413; P. LE Roux et A. TRANOY, Rome et les indigènes dans le Nord-Ouest de la péninsule ibérique. Problèmes d'épigraphie et d'histoire, $M C V$, IX, 1973, pp.178-179 et 225-226.

86 P. LE Roux et A. TRANOY, Villes et fonctions urbaines dans le Nord-Ouest ibérique sous domination romaine, Portugalia, IV-V, 1983-1984, p.207.

87 P. LE Roux, Municipium Latinum et municipium Italiae: à propos de la lex Irnitana, dans Actes du colloque en mémoire de A. Degrassi (Rome, 1988), Rome, 1991, pp.565-582; J. MUÑIZ COELlO, La política municipal de los Flavios en Hispania. E1 municipium Irnitanum, SHHA, II-III, 1, 1984-1985, pp.151-176. 
mantica (Vettonnie nord-occidentale) ${ }^{88}$. Rien n'indique qu'une telle réorganisation territoriale ait touché toute la Vettonnie mais P. Le Roux écrit, à propos de ces cippes augustéens: "Ils émanent tous de Lusitanie et leur datation, lorsqu'elle est décelable, se place autour de 5/6p.C., qui semble donc avoir été une année décisive pour la mise en place des limites des cités» ${ }^{89}$. On comprendra aisément qu'il était alors sans doute trop tôt pour accorder des privilèges juridiques aux cités vettonnes.

L'octroi du ius Lati a sans doute été l'occasion de modifications institutionnelles difficiles à percevoir mais surtout, a permis à l'aristocratie urbaine, d'accéder à la citoyenneté romaine. La mention de la tribu Quirina à Capera mais aussi à Caesarobriga, n'est pas tant l'indice d'une municipalisation que de l'octroi du droit latin à des individus qu'il fallait bien enregistrer dans une des tribus ${ }^{90}$. En outre, la mention de la tribu étant peu répandue dans les structures nominales des Vettons citoyens romains (eux-mêmes peu nombreux), on peut en déduire que l'octroi du ius Latii aux Vettons, puis la promotion municipale de certains oppida n'a pas été d'un très grand impact en Vettonnie. M. Salinas est d'avis que l'œuvre municipale des Flaviens a été faible dans la mesure où la mention de la tribu Quirina indique que la citoyenneté n'a été obtenue que postérieurement au beneficium de Vespasien: les documents épigraphiques mentionnant la tribu Quirina peuvent fort bien dater du début du Ilème siècle ap. J.C., surtout si on considère l'attention toute particulière qui fut accordée à l'entretien des voies de

88 CIL II, 857, 858, 859 et 5033; C. MoRÁn, Epigrafía salmantina, Salamanque, 1922, p.48-49; J. MALUQUER, op.cit., p.140 n. ${ }^{\circ} 165$; d'autres bornes de ce type ont été retrouvées en Lusitanie: entre les Talabrigenses et les Lancobrigenses (HAE, 1442), et entre deux peuples limitrophes dans la serra de Caramulo $(A E, 1954,88)$; dans ce dernier cas, nous connaissons le nom du gouverneur de Lusitanie qui est à l'origine de l'opération: Q. Articuleius Regulus qui a sans doute également procédé au bornage des territoires vettons puisque tous ces documents sont plus ou moins contemporains.

89 P. LE Roux, Cités et territoires: l'épigraphie des limites, $M C V$, XXX, 1994, p. 40 .

90 P. LE Roux, art.cit., p.41; sur la tribu Quirina, cf. R. KNOX MAC ELDERRY, Vespasian's reconstruction of Spain, JRS, VIII, 1918, p.68; H. GALSTERER, Untersuchungen zum rômischen Stadtewesen aufder iberischen Halbinsel (MF, VIII), Berlin, 1971, p.46; A. Tranoy, La Galice romaine. Recherches sur le nord-ouest de la péninsule ibérique dans VAntiquité, Paris, 1981, p.202; E. WIEGELS, op.cit., p.6; G. ALFOLDY, Rômisches Stadtewesen aufder neukastilichen Hochebene. Ein Testfall fur die Romanisierung, Heidelberg, 1974, p.30. 
la région par des empereurs tels que Trajan ou Hadrien ${ }^{91}$. Cependant, cette prise de position peut être nuancée si on considère qu'il ne faut pas automatiquement lier intérêt porté aux infrastructures et aux villes, et promotion municipale. La preuve en est l'œuvre d'Auguste en matière de délimitation de territoires et d'urbanisme, sans aucune œuvre municipale en Vettonnie ${ }^{92}$.

Ainsi, le réseau administratif vetton, d'après ce que les rares sources nous suggèrent, devait fonctionner, depuis la fin du ler siècle, sur plusieurs niveaux:

- Il est probable qu'un grand nombre de noyaux de peuplement mineurs ne s'est vu accorder ni ius Latii ni statut municipal, n'étant pas reconnu par les autorités romaines comme entités autonomes ${ }^{93}$. Ces noyaux secondaires devaient dépendre de centres plus importants. Les institutions de ces noyaux (oppida?) devaient être très rudimentaires (les magistrati de Las Merchanas?) en raison de leur grande dépendance administrative.

- Turgalium semble avoir été un cas particulier. Aucune institution municipale, aucun statut privilégié n'y apparaissent. Cependant, on ne peut ranger cette ville dans la catégorie précédente car Turgalium était en fait une préfecture d'Emerita Augusta, c'est-à-dire un territoire sous le contrôle administratif et économique direct de la capitale provinciale voisine. La création de cette préfecture a pu être contemporaine de la naissance même d'Emerita Augusta (25 av. J.C.) ${ }^{94}$.

91 M. SALINAS, Las ciudades romanas de Lusitania oriental: su papel en la transformación del territorio y la sociedad indígena, dans Les villes de Lusitanie romaine. Hiérarchie et territoires. Table ronde internationale du CNRS (Talence, 1988), Paris, 1990, p.261.

92 E. García FernándeZ, El ius latii y los municipia latina, SHHA, IX, 1991, pp. 29-41 fait cependant remonter à Auguste l'apparition des premiers municipia latina ainsi que l'association droit latin / statut municipal.

93 J. MuÑIZ COELlo, La política municipal de los Flavios en Hispania. El municipium Irnitanum, SHHA, II-III, 1, 1984-85, p.158, indique que «nombre d'entités indigènes sont restées en marge de l'édit de Vespasien, ne possédant pas un minimum d'organisation urbaine susceptible d'être modelé comme cadre de fonctionnement municipal».

94 Hyginus, Corpus agrimensorum Romanorum, I, 136; P. GUICHARD, art.cit., p.69: "Les communautés les plus frustes sont les plus atteintes et, certaines d'entre elles sont placées sous contrôle direct des colonies et quasiment intégrées à leur territoire sous forme de préfectures comme les praefecturae Mullicensis et Turgaliensis d'Emerita»; sur le statut juridique des préfectures à’Emerita, cf. P. LÓPEZ PAZ, Obser- 
- L'échelon supérieur était sans doute représenté par les oppida Latina, noyaux plus considérables qui ont bénéficié $\mathrm{du}$ droit latin mais sans pour autant avoir accédé au rang municipal. Ces centres exerçaient une autorité administrative plus ou moins directe sur les sites précédents et étaient peut-être à la tête de vastes civitates, étant entendu que toutes les capitales de civitates n'étaient pas des municipes. Ces noyaux n'étaient cependant pas autonomes sur le plan administratif mais relevaient directement des autorités provinciales, voire des municipia. Dans cette catégorie des oppida latina, on peut ranger Bletisa, Augustobriga et peut-être Las Merchanas, Mirobriga et Salmantica. Sur le plan institutionnel, l'épigraphie atteste l'existence d'un senatus à $A u$ gustobriga, de decuriones à Mirobriga, d'un ordo à Salmantica.

- Enfin, les municipes étaient des cités de droit latin qui avaient accepté un statut d'autonomie plus important, en vertu d'une loi municipale dont on n'a malheureusement retrouvé aucun exemplaire en Vettonnie $^{95}$. Ces villes étaient pour la plupart de petite dimension et leur population peu nombreuse. Elles n'en étaient pas moins à la tête de territoires parfois très vastes et exerçaient une fonction d'intermédiaire entre les autorités romaines et la population. Elles n'étaient sans doute pas obligatoirement des chefs-lieux de civitates, même si c'était certainement le cas le plus répandu. Capera, Caesarobriga et peut-être Salmantica ont bénéficié de cette promotion administrative et juridique, tout comme les deux Lancia (Lancia Transcudana et Lancia Oppidana) si on admet que la restauration du pont d'Alcántara a été l'occasion de la promotion municipale des communautés ayant participé à cette œuvre. Sur le plan institutionnel, ces municipes se caractérisaient par l'existence de magistratures municipales qui n'auraient pas déparé dans un contexte italien: duumvires, quaestores, aediles, praefecti fabrum, flaminicae perpetuae...96.

Ainsi, il serait erroné de voir dans le réseau administratif vetton un système parfait de cadres s'emboîtant parfaitement les uns dans les

vaciones sobre el concepto y estatuto jurídico de las praefecturas. El ejemplo de Emerita Augusta, dans II Congresso peninsular de Historia Antiga (Coimbra, 1990), Coïmbre, 1993, pp.741-751.

95 Le contenu de ces lois pouvait avoir des caractères communs avec celui de la lex Irnitana: $c f$. A. D'ORs, La ley flavia municipal, AHDE, IV, 1984, pp.535-573.

96 R. ETIENNE, op.cit., p.167, explique l'adjectif prima devant perpetuae flaminica par le fait que Caesarobriga venait alors d'accéder au rang municipal. 
autres, ou de cercles concentriques. Le système d'encadrement romain au niveau infra-provincial était infiniment plus complexe, tenant compte des particularités (attitude au moment de la conquête, présence de richesses minières, localisation stratégique...) pour créer un réseau inextricable de statuts, débordements frontaliers et particularismes. Il serait vain, surtout en l'absence de sources nombreuses et explicites, de vouloir restituer dans le détail cette organisation administrative qui devait en grande partie s'appuyer sur les structures préexistantes.

$\mathrm{Au}$ terme de cette étude, nous sommes en mesure de fournir la liste d'une douzaine de noyaux de peuplement qui ont dû être des chefs-lieux et donc posséder des institutions propres:

- En Vettonnie du nord: Avela, Salmantica, Mirobriga, Bletisa, Polibeda, Lancia Oppidana, Lancia Transcudana et peut-être Valuta et Vrunia.

- En Vettonnie centrale: Caesarobriga, Augustobriga, Capera

- En Vettonnie du Sud: Turgalium, Lacimurga.

Il s'agit-là d'une liste non exhaustive qui ne doit pas faire oublier que d'autres sites vettons, cités par Ptolémée, hypothétiquement urbains, n'ont pas été du tout localisés \{Lama...) et pouvaient constituer des chefs-lieux de civitates, tout comme deux de ces quatorze toponymes ont pu se situer sur une même circonscription, en remplissant des fonctions différentes ou complémentaires. Ceci n'est d'ailleurs pas un obstacle au fait que chacun pouvait posséder ses propres institutions. En effet, nous pensons que la Vettonnie romaine ne devait pas se présenter sous la simple forme d'un ensemble de territoires ruraux (des civitates même si le terme apparaît peu dans l'épigraphie, ce qui est sans doute significatif) dominés chacun par une ville, chef-lieu administratif, marché économique, centre de romanisation. $\mathrm{La}$ réalité devait être moins simple. L'étude de l'organisation administrative laisse plutôt deviner qu'un même territoire pouvait comprendre plusieurs noyaux importants de peuplement, que le plus vaste n'était pas obligatoirement le plus privilégié sur le plan juridique, que l'influence et le rôle de chacun de ces noyaux aient été différents sur les plans administratif et économique: l'idée du voisinage d'un gros bourg rural, principal foyer de peuplement aggloméré et centre de consommation, et d'une ville n'ayant qu'un rôle de chef-lieu administratif, ne nous semble pas devoir être écartée. Dans l'extrême sud de la Vettonnie, le seul noyau de peuplement digne de ce nom est Lacimurga mais l'archéologie est loin de 
démontrer son caractère urbain et l'épigraphie est totalement muette quant à ses institutions ou son statut juridique. En outre, il est probable que les termes de vicus et de castellum, que l'on retrouve avec Vicus Caecilius (région de Puerto de Béjar) et Castellum Ciseli (région de Talavera de la Reina?), sont d'autres traductions de cette grande hétérogénéité en matière administrative ${ }^{97}$.

L'exemple de la Vettonnie est donc assez révélateur d'une certaine politique pragmatique des Romains en matière d'organisation administrative des territoires conquis. Loin d'avoir été négligé ou l'objet d'une politique d'uniformisation forcée, le territoire vetton présente l'aspect, sous la domination romaine, d'un espace relativement harmonieux, où furent respectés à la fois l'unité ethnique de son peuplement et, sans doute, le manque d'unité de son organisation avant la conquête romaine. Il est vrai, en outre, que les Vettons n'ont jamais constitué un problème pour les autorités romaines, sans doute en raison de la faiblesse de leur peuplement, de leur faible développement socio-économique, de l'absence d'unité politique et de résistance armée prolongée à l'encontre des Romains. Malgré les modifications indispensables à tout territoire conquis (fondation de villes neuves, création d'un réseau routier, développement de villae...), les Romains n'avaient guère de raisons de bouleverser le pays vetton, et ce d'autant plus que ce dernier n'a jamais constitué pour eux un espace réellement attractif.

97 P. LE Roux, Vicus et castellum en Lusitanie sous l'Empire, dans El medio rural en Lusitania romana. Formas de hábitat y ocupación del suelo. Actas de la Mesa Redonda Internacional (Salamanca, 1993) (SHHA, X-XI), Salamanque, 1994, pp.154.

Conimbriga, 40 (2001) 5-35 
FIG. I.

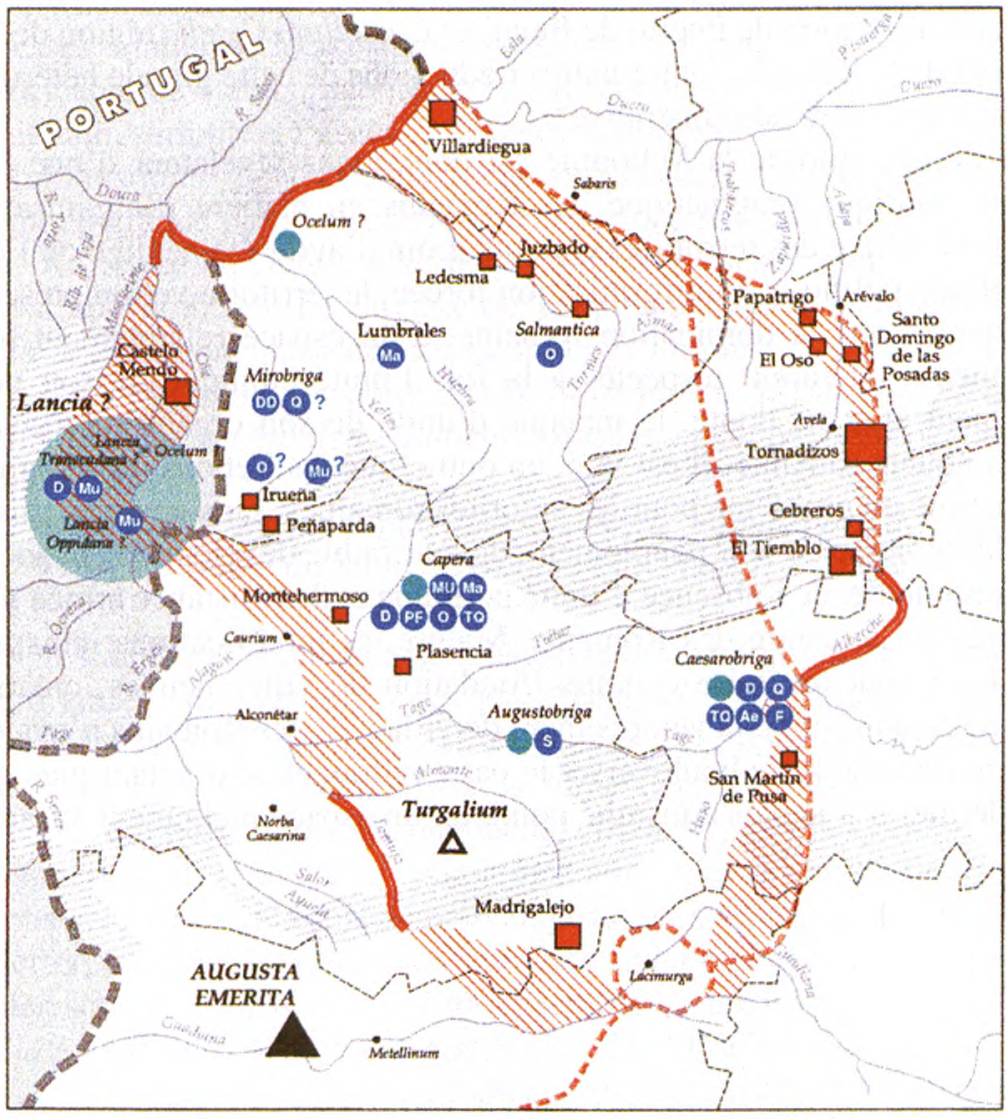

Limite ethrique probable du pays vetton

NIV Limite ethnique non localisée

Noyau de peuplement vetton
mentionnés par Pline en tant que cuté stipendiaire

Témoignages épigraphiques :

Verracos periphériques en pays vetton

$a^{e^{x^{2}}} a^{x^{2}}{ }^{2}$

a - s Fronticre (approximative) de la Lusitarie

$x=\infty$
$n=1$ Modification de frontiere provinciale
entre Pline et Ptolemee

Capitale provinciale et de contentu. 
FIG. II

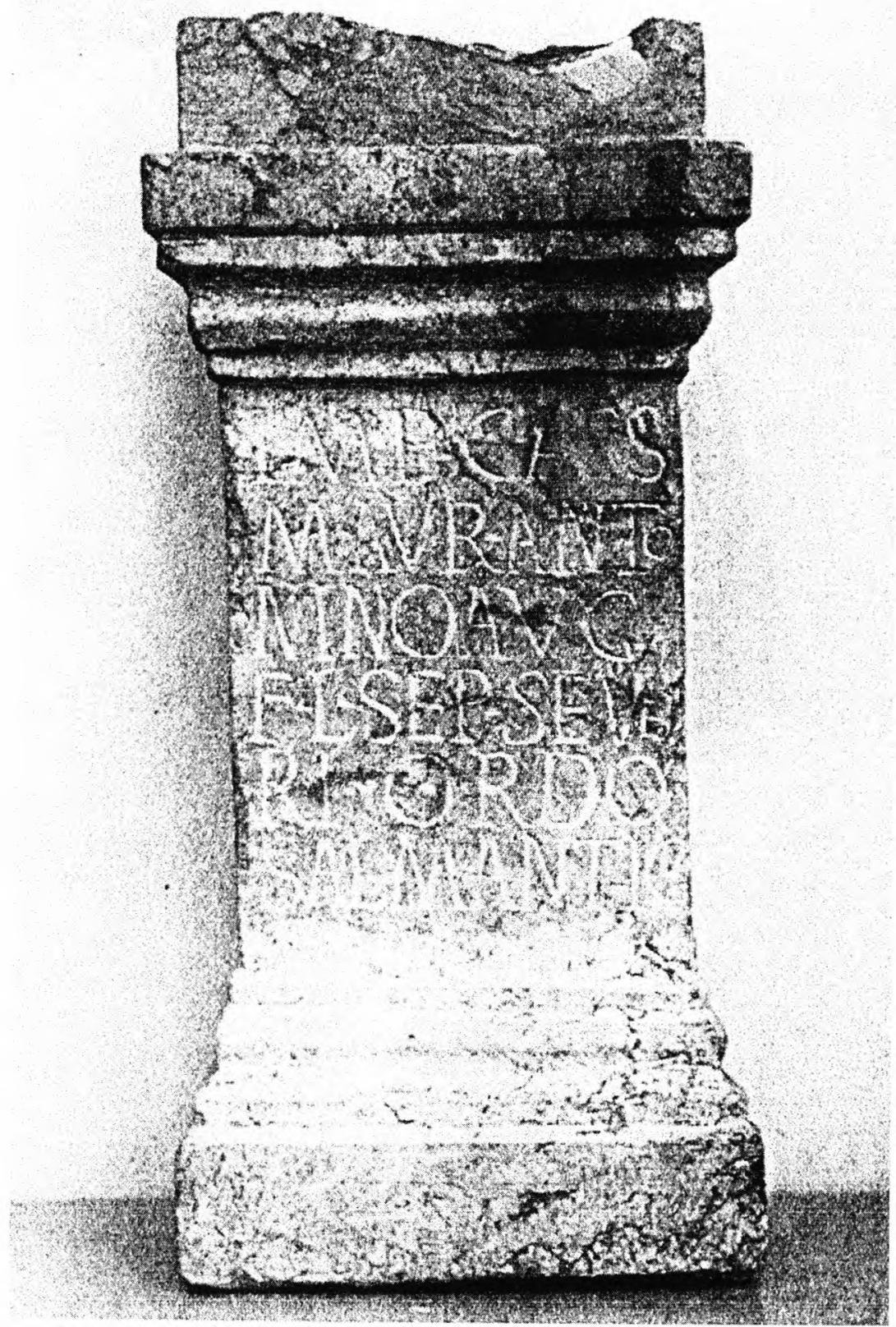


FIG. III

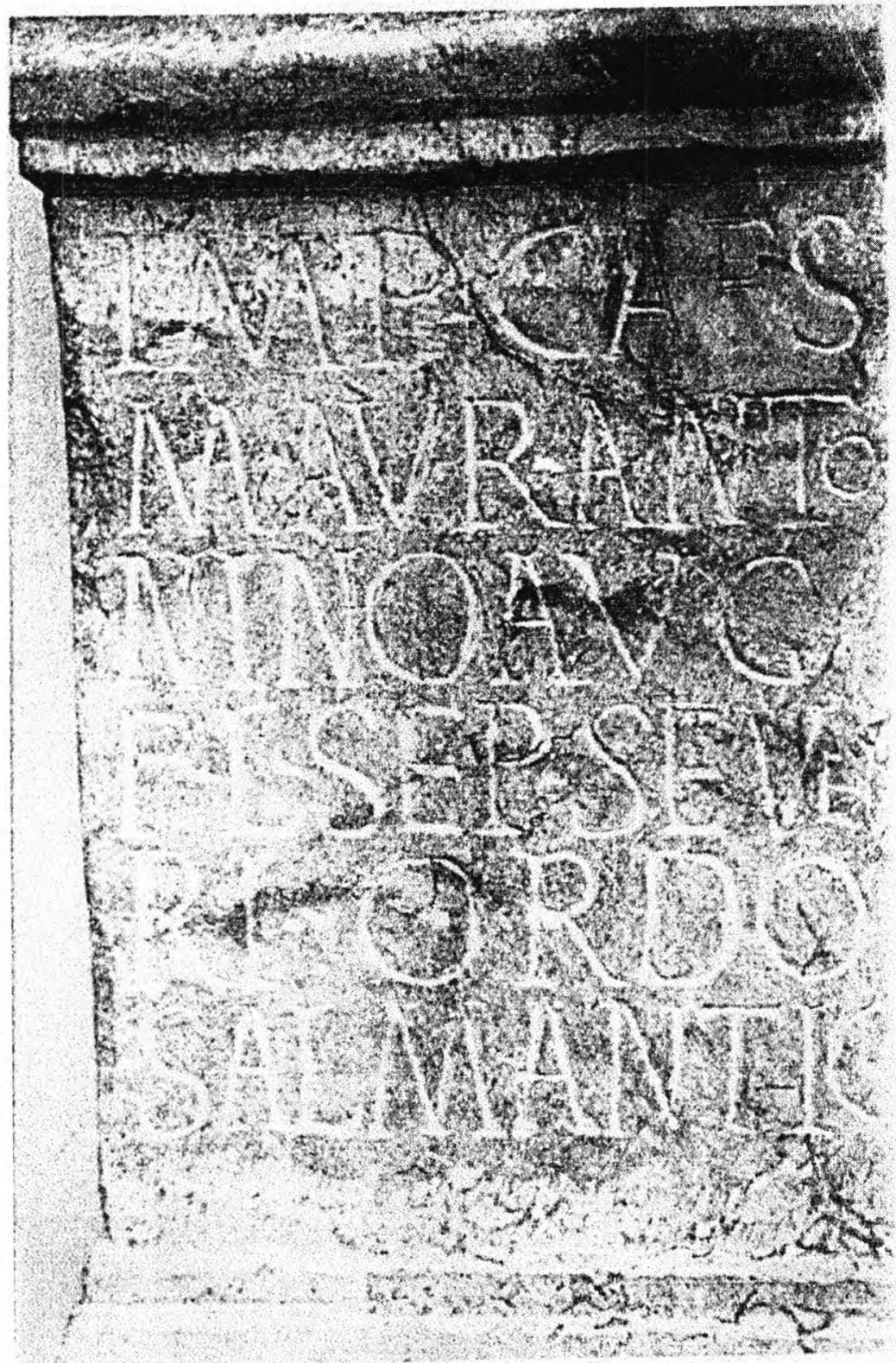

